\title{
Hydraulic Infinite Linear Actuator - The Ballistic Gait Digital Hydro-Mechanical Motion
}

\author{
Martin Hochwallner and Petter Krus \\ Division of Fluid and Mechatronic Systems, Linköping University, Linköping, Sweden \\ E-mail: martin.hochwallner@liu.se
}

\begin{abstract}
The Hydraulic Infinite Linear Actuator, HILA, has been presented in [1], [2], and [3]. The novel actuator consists of one, two or more double acting cylinders with a common piston rod and hydraulically detachable pistons. In the basic gait [1], alternatingly, one cylinder engages and drives the load while the other retracts, the HILA thus works in a kind of rope climbing motion. But the concept allows also other gaits, pattern of motion.

This contribution focuses on the ballistic gait, a pattern of motion where one cylinder engages to give the load a push. Then the load carries on with its motion by inertia, cylinders disengaged. The actuator realizes thus hydro-mechanical pulse-frequency modulation (PFM).

This gait is energy efficient and able to recuperate energy.
\end{abstract}

Keywords: novel actuator, infinite linear motion, digital fluid power, digital hydromechanical motion, energy recuperation

\section{Introduction}

The gait of the Hydraulic Infinite Linear Actuator, HILA, Figure 1 , where alternatingly one cylinder disengages to retract has been studied and presented in for example [1]. This gait is characterized by, that all time at least one cylinder is engaged to drive the load, Figure 3a. A smooth, high performance motion can be realized by this actuator in this gait. Gaits define pattern of motion. HILA can be operated in various gaits, whereby gaits are suitable for various situations and shall be used in together in an application to achieve optimal performance. This contribution presents another gait, the ballistic gait, Figure 3b, where temporarily all cylinders are disengaged to allow some kind of freewheeling exploiting the inertia of the load.

Conventional hydraulic linear actuators, i.e. cylinders, are common and mature components of hydraulic systems. Ongoing research focuses on secondary control with multi- chamber cylinders [5], various concepts of digital hydraulics [6], individual metering [7], advanced control concepts [8,9], and sensor-less positioning with stepper drives [10].

In [11] presented Gall and Senn a linear hydraulic drive exploiting the inertia of the load in combination with so called freewheeling valves for saving energy [6]. In the ballistic gait, this basic idea is applied on HILA. The freewheeling valves allow motion without discharging flow from the supply line. The ballistic gait goes one step further and decouples the full hydraulics, even the cylinder, from the load and thus eliminates losses in the hydraulics and friction losses in the cylinder. The drawback is the necessity of retracting the cylinder. In this contribution, the simple but inefficient way of using the supply flow is applied. Research on effective alternatives is necessary.

The actuator can be operated solely in the ballistic gait, but the highest benefit is expected in systems combining gaits.

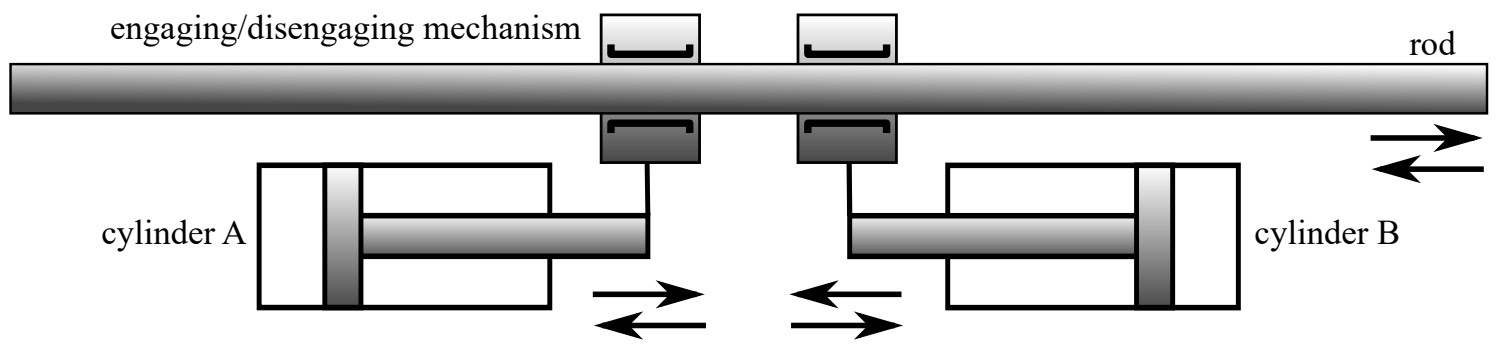

Figure 1: HILA: Two double-acting cylinders temporarily engage/disengage to the common rod to driving the load. The load may be attached to the rod or to the actuator. 


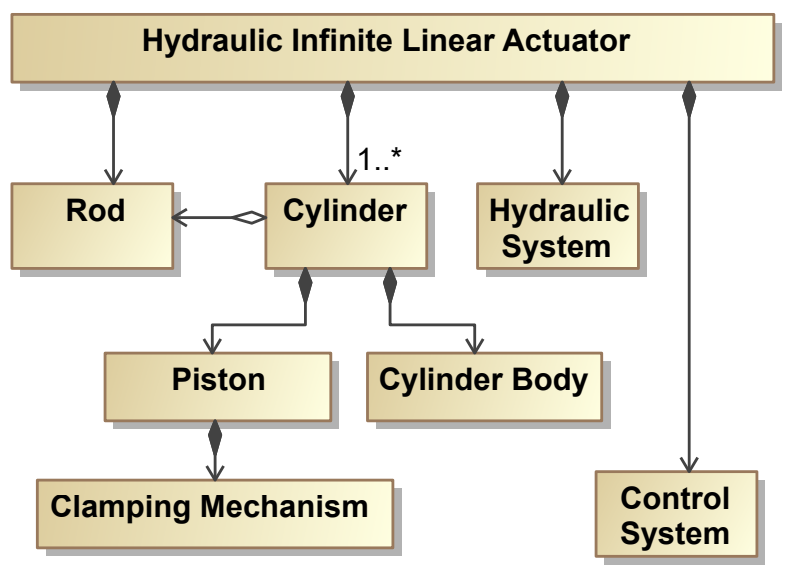

Figure 2: Structural breakdown as SysML Block Definition Diagram [4].

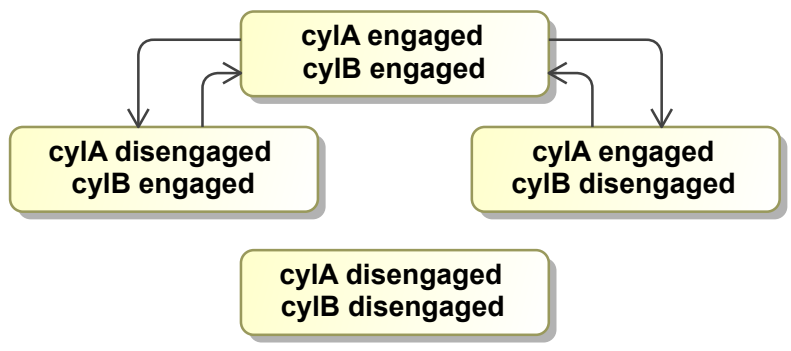

(a) The basic gait.

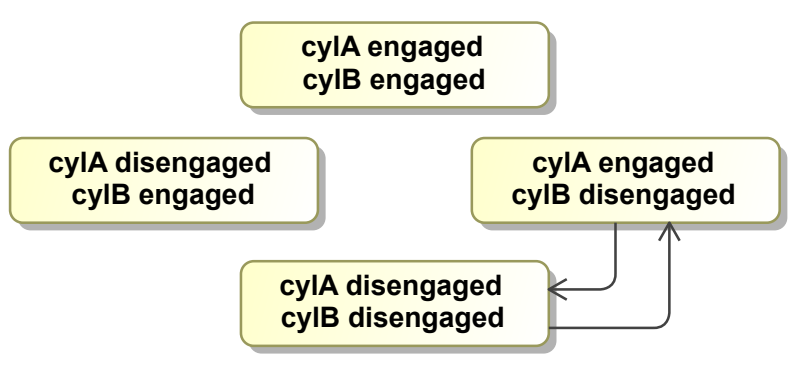

(b) The ballistic gait.

Figure 3: Gaits as Activity Diagram.

For example, machines may require the following working modes and thus gaits. A high performance, high accuracy working stroke with medium to infinite stroke length applying the basic gait. A short high force work stroke, where the force and stiffness of two cylinders are added up. A holding period utilizing the infinite hydraulic stiffness presented in [2] where no power is required. And a fast and efficient not-work stock for retraction realized by the ballistic gait. One application field could be mounting plates of machines for advanced injection molding.

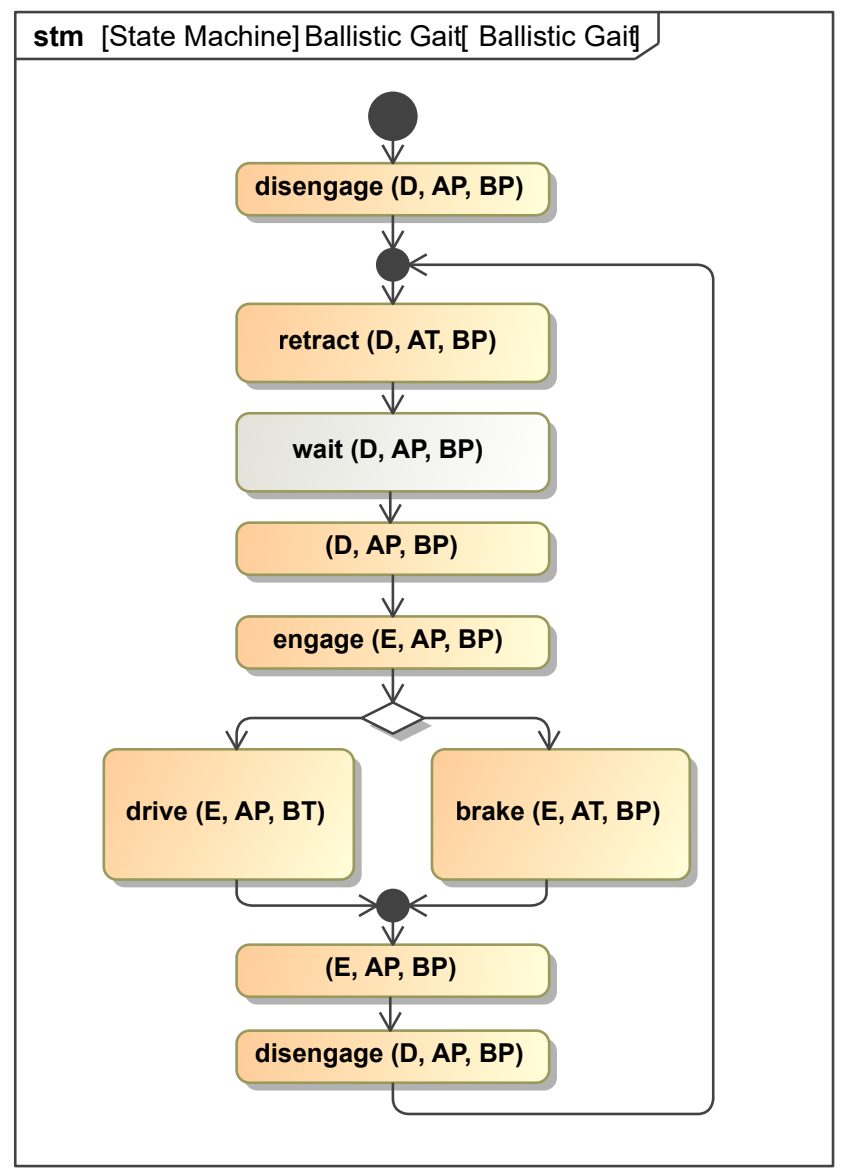

Figure 4: Control sequence depicted as SysML State Machine [4].

The controller outputs are stated in braces: E: engaged, D: disengaged, AP, AT, BP, AT: chambers: A, B; pressure levels: $P$ : supply, T: tank.

\section{Basic Concept}

The control concept is shown in Figure 4 and the corresponding schematics of the hydraulic circuit in Figure 6. To move the load, first the cylinder retracts to the retracted position. An end-position cushion is used to stop the cylinder. The cylinder does not drive any load beside the piston assembly and so the kinematic energy is low. After waiting for the engaging condition both valves are switched fully to the high pressure side so that the motion of the cylinder is not locked. Now the cylinder engages and the increasing friction in the clamping mechanism accelerates the piston to the velocity of the rod. By switching the valve of chamber B to the low pressure side, force is applied to accelerate the load. If instead, the valve of chamber $\mathrm{A}$ is switching to the low pressure side a force to deaccelerate the load is applied. When the cylinder reaches the end of driving stroke, both valve are switched to the high pressure side and so the rod drives the cylinder. Then, the cylinder disengages, before the piston hits the end-position cushion. To repeat the cycle the cylinder retracts to the retracted position. 


\begin{tabular}{lcr}
\hline effective load force & $F_{\mathrm{L}}$ & $0 \mathrm{kN}$ \\
effective mass / inertia & $m_{\mathrm{L}}$ & $50 \mathrm{t}$ \\
effective damping & $b_{\mathrm{L}}$ & $2000 \mathrm{~N} \mathrm{~s} / \mathrm{m}$ \\
\hline supply pressure & & $250 \mathrm{bar}$ \\
tank pressure & & $10 \mathrm{bar}$ \\
\hline
\end{tabular}

Table 1: Parameters for the load and system in simulation

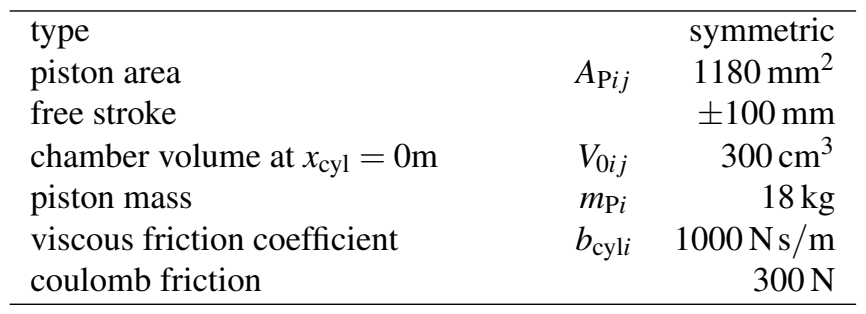

Table 2: Parameters for the cylinders in simulation

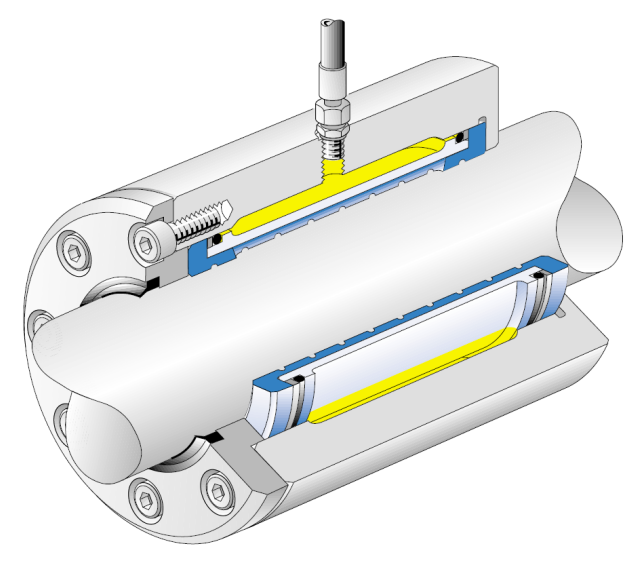

Figure 5: Hydraulic hub-shaft connection Octopus from ETP Transmission $A B$ [12]

\section{System}

Figure 1 shows the schematic of the actuator. The actuator may drive a load attached to the rod or to the actuator itself (the rod is stationary). The assumed load consists of inertia, i.e. a mass $m_{\mathrm{L}}$, and additional force $F_{\mathrm{L}}$, and viscous friction. The parameters for the simulation are presented in Table 1 and 2.

The engaging / disengaging subsystem is the enabler for HILA. One component which can realize the engaging / disengaging subsystem is the COTS hydraulic hub-shaft connection [12], see Figure 5. The hub-shaft connection engages due to the hydraulically actuated membrane being pressed against the rod so that the friction between membrane and rod transfers the cylinder force. To actuate the hub-shaft connection the fast switching valve FSVi 4.1 [13] from Linz Center of Mechatronics $\mathrm{GmbH}$ is used. This system can fully engage and disengage within $10 \mathrm{~ms}$.

Two exemplary hydraulic schematics to drive the cylinders are shown in Figure 6 and 7. The schematic in Figure 6 shows a system using one fast proportional valve per cylinder chamber and resemble thus an independent-metering valve.

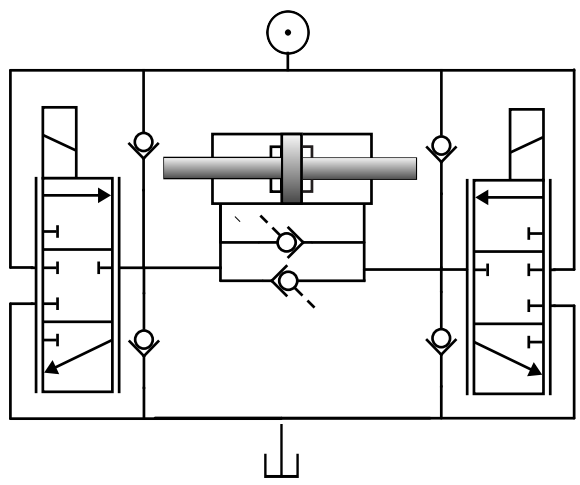

Figure 6: Schematic, system P, with independent-metering valve, one cylinder.

Independent-metering valve, modeled as one individual proportional valve per chamber. Four check valves protect against over pressure and cavitation. Two lockable check valves allow cross flow when required. Table 2 and 3 show the parameter.

\begin{tabular}{lcr}
\hline nominal flow at 35 bar per edge & $q_{\text {nom }}$ & $25 \mathrm{~L} / \mathrm{min}$ \\
corner frequency for small & $f_{\mathrm{V}}$ & $200 \mathrm{~Hz}$ \\
amplitudes & & \\
damping & $\delta$ & 0.7 \\
actuating time for signal step 0 to 1 & $T_{\mathrm{V} \text { step }}$ & $5 \mathrm{~ms}$ \\
\hline
\end{tabular}

Table 3: Parameters for the Proportional Valve in Simulation

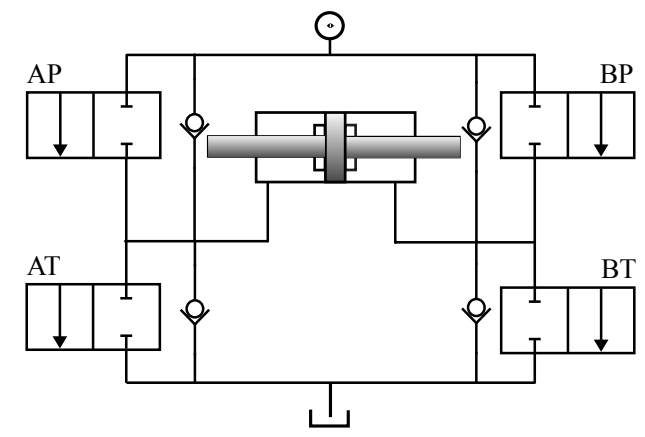

Figure 7: Schematic, system D, with four fast on/off valves, one cylinder.

A candidate for the on/off valves is the fast switching multi poppet valve from the Linz Center for Mechatronics (LCM) $\mathrm{GmbH}$, [14]. Four check valves protect against over pressure and cavitation. Table 2 and 4 show the parameter.

\begin{tabular}{lcr}
\hline nominal flow at 5 bar & $q_{\text {nom }}$ & $85 \mathrm{~L} / \mathrm{min}$ \\
opening / closing time & $T_{\mathrm{V}}$ & $2 \mathrm{~ms}$ \\
\hline
\end{tabular}

Table 4: Parameters for the On/Off Valve in Simulation. The parameters are based on th fast switching multi poppet valve from the Linz Center for Mechatronics (LCM) GmbH, [14].

Therefor, this system can be used to implement other gaits, for example for smooth motion as presented in [1]. Applications may require combining various gaits to fulfill their requirements. The system is dimensioned as an ordinary hydraulic 
servo system to facilitate position control and not for high velocity. Additional to their protective function against overpressure and cavitation, the check-valve provide additional flow paths and thus increase the efficiency and reduce the requirements on the proportional valves. A capable check-valve design is presented in [10]. For this concept the proportional valves are used as three position on/off valves. The three positions are: $\mathrm{P}$, when the cylinder chamber is connected to the supply pressure; $\mathrm{T}$, when connected to the tank pressure; and off, when the valve is closed.

A second concept, fully digital, is shown in Figure 7. In cases where other gaits are required this concept can be combined with for example hydraulic switching technology as the Hydraulic Buck Converter [6], or it cam be equipped with parallel proportional valves resembling the system in Figure 6. In the level of detail as this contribution goes, the mayor difference between this two systems is the valve size. The on/off valves have 9 times the nominal flow rate of one edge of the proportional valves and produces thus only $1 \%$ of the pressure drop. This system is dimensioned for high flow rates and thus high velocity. The additional flow path through the check valves is thus not relevant.

\section{Simulation Results}

Figures 8 and 9 show simulation results of the two presented systems. In both cases, the actuator accelerates the load, then keeps the velocity beyond the desired velocity, and after a few strokes the actuator deaccelerates the load to stop, whereby it recuperates energy. The systems go for different desired velocities. Although both systems and thus both simulation results are similar, the different working conditions emphasize different aspects of the concept. Some aspects appear when comparing the two results. Both systems use only one cylinder.

Plot 1 and 2 show the position of the load and the cylinder. The cylinder retracts, then thrusts the load, and then repeats.

While retracting, the velocity is limited to $-0.65 \mathrm{~m} / \mathrm{s}$, and $-5.2 \mathrm{~m} / \mathrm{s}$ respectively by the pressure drop through the fully opened valve. Plot 6 , chamber pressure, for the system $\mathrm{P}$, shows that in the load pressure, the difference between the chamber pressures, is small while retracting as it only covers the cylinder friction. For system $\mathrm{D}$ the force is a bit higher as the velocity is higher. As the retraction is much faster details can not be seen in the plot. Plot 5, $F_{\text {cyl }}$, does not include cylinder friction as it shows the force of the cylinder applied on the load, i.e. the force passing the engaging/disengaging mechanism.

During the acceleration phase the thrusts take less and less time as the velocity increases. Especially in the case of system $P$, decreases the force with increasing velocity as the pressure drop in the valves increase. With system $\mathrm{D}$, this effect is small for the shown velocity. As shown in plot 4, supply flow, the needed flow is identical to a conventional cylinder, blue area, orange dashed line, but there is additional flow used for retraction, green area.

When a velocity beyond the desired velocity is reached, the actuator waits with the next thrust for the velocity to fall below the desired. For that time in contrast to a conventional cylinder no flow is needed, area below the orange dashed line. Therefor energy is saved.

In the deacceleration phase, chamber $A$ instead of $B$ is switched to the low pressure side and thus the force direction inverted. Now the cylinder drives the flow through the valve and thus the pressure drop through the valve increases the load pressure. Therefor the force and thus the acceleration is higher in the deacceleration than in the acceleration phase. The pressure drop and thus the force decreases with falling velocity. During this phase the check-valves provide an additional flow path and therefore, the pressure drop through the valve is much smaller as in the acceleration phase. The flow direction is reversed and thus the oil drawn from the tank and delivered into the supply line. Hence, energy is recuperated. As the flow from the tank has to pass trough the valves, boosted tank pressure is necessary, see Table 1 . The check-valves improve the efficiency, especially in system $P$. The valves in system D are sufficient big so that check-valves influence in minor.

\section{Analysis}

The thrust stroke length $L_{\mathrm{T}}$ of a full thrust is constant, ignoring second order effect. Therefor, the energy added to the load is:

$$
E_{\mathrm{T}}=F_{\mathrm{cyl}} L_{\mathrm{T}}
$$

whereby the cylinder force $F_{\text {cyl }}$ can be calculated as:

$$
F_{\text {cyl }}=\left(p_{\mathrm{A}}-p_{\mathrm{B}}\right) A-F_{\text {losses }}
$$

This is also the energy removed from the load while braking one full thrust.

Ignoring the friction losses and the pressure drop through the valves the nominal cylinder force $F_{\text {cylN }}$ is calculated as:

$$
F_{\text {cylN }}=\left(p_{\mathrm{S}}-p_{\mathrm{T}}\right) A
$$

and the nominal energy added per full thrust is

$$
E_{\mathrm{TN}}=\left(p_{\mathrm{S}}-p_{\mathrm{T}}\right) A L_{\mathrm{T}}
$$

For system D the nominal values represent the system well. The further analysis assumes a system which can sufficiently approximated by this nominal system, i.e. a system like system D.

The velocity of the load increases from the velocity before the thrust $v_{0}$ to the velocity after the thrust $v_{1}$ according to:

$$
v_{1}=\sqrt{v_{0}^{2}+\frac{2}{m}\left(E_{\mathrm{T}}-E_{\mathrm{L}}^{*}\right)}
$$

whereby $E_{\mathrm{L}}^{*}$ stands for the reduction of kinetic energy of the load due to load forces and friction in the duration corresponding to $E_{\mathrm{T}}$.

The trend of the velocity during acceleration phase can thus be approximated by the following equation, where $k$ is the 

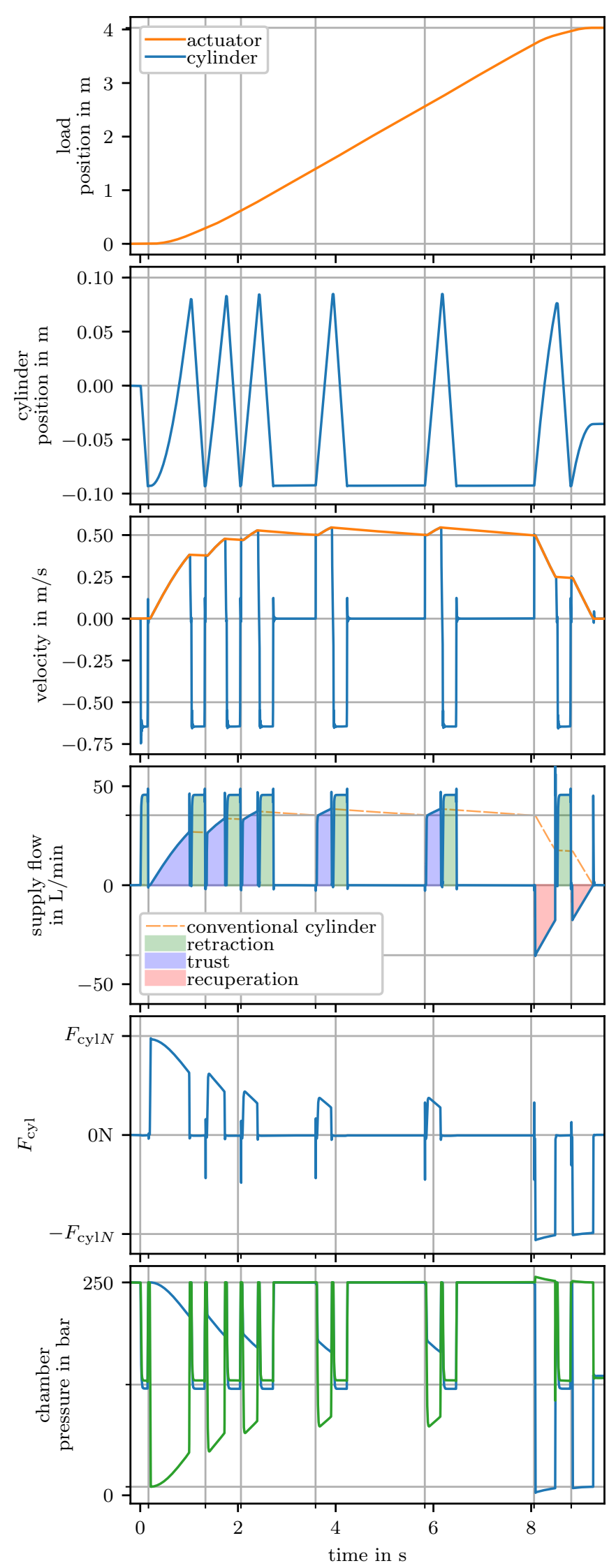

Figure 8: Simulation results system P: two proportional valves / independent metering,

Schematic Figure 6. The desired velocity is $0.5 \mathrm{~m} / \mathrm{s}$.
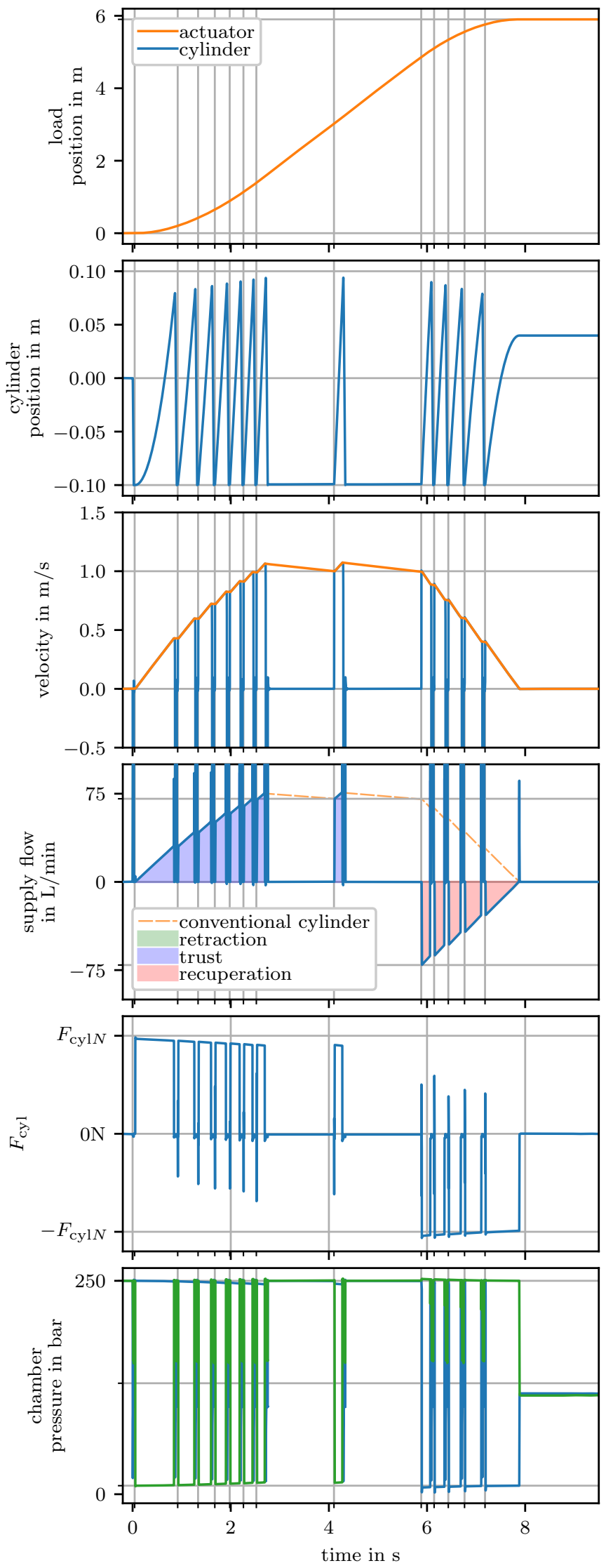

Figure 9: Simulation Results system D: on/off valves, Schematic Figure 7. The desired velocity is $1 \mathrm{~m} / \mathrm{s}$. 
number of thrusts. Losses, friction and external forces are ignored.

$$
v_{k}=\sqrt{\frac{2}{m} E_{\mathrm{TN}} k}
$$

This trend can be seen in Figure 9, where the velocity after four thrusts is twice as high as after one.

During the keeping velocity phase the actuator has to counteract the reduction of kinetic energy of the load by external forces and friction by recurring thrusts. Assuming a quasiconstant load force $F_{\mathrm{LX}}$, considering external forces and friction, leads to the relation:

$$
F_{\text {cyl }} L_{\mathrm{T}}=E_{\mathrm{T}}=E_{\mathrm{L}}^{\#}=F_{\mathrm{LX}} L_{\mathrm{C}}
$$

whereby $L_{\mathrm{C}}=L_{\mathrm{T}}+L_{\mathrm{B}}$ and thus:

$$
\frac{F_{\text {cyl }}}{F_{\mathrm{LX}}}=\frac{L_{\mathrm{C}}}{L_{\mathrm{T}}}=1+\frac{L_{\mathrm{B}}}{L_{\mathrm{T}}}=\frac{T_{\mathrm{C}}}{T_{\mathrm{T}}}
$$

The undesired variation of the velocity during the keeping velocity phase can be characterized with the absolute variation $\Delta v$ and the relative variation $r$ of the velocity, which can be expressed as:

$$
\begin{gathered}
\Delta v=v_{1}-v_{0}=\sqrt{v_{0}^{2}+\frac{2}{m}\left(E_{\mathrm{T}}-E_{\mathrm{L}}^{*}\right)}-v_{0} \\
\leq \widehat{\Delta v}=\sqrt{v_{0}^{2}+\frac{2}{m} F_{\mathrm{cyl}} L_{\mathrm{T}}}-v_{0} \\
r=\frac{v_{1}-v_{0}}{v_{0}}=\sqrt{1+\frac{2\left(E_{\mathrm{T}}-E_{\mathrm{L}}^{*}\right)}{m v_{0}^{2}}}-1 \\
\leq \widehat{r}=\sqrt{1+\frac{2 F_{\mathrm{cyl}} L_{\mathrm{T}}}{m v_{0}^{2}}}-1
\end{gathered}
$$

For the limits it is assumed that $E_{\mathrm{L}}^{*}>0$ and always reduces the kinematic energy of the load, i.e. friction.

Both, the absolute variation and the relative variation of the velocity gets smaller with increasing velocity.

\subsection{Scaling, Limitations and Design Estimations}

In walking mechanics [16], the Froude number, $\mathbf{F}=\frac{v^{2}}{l g}$, where $v$ is the velocity, $l$ the leg length, and $g$ gravity, is used to characterize the transition from walking to running. Remarkable, the inner fraction in Equation 10b has the same structure and the following can be derived.

$$
\mathbf{F}=\frac{v_{0}^{2} m}{L_{\mathrm{T}} F_{\mathrm{cyl}}}=\frac{v_{0}^{2}}{L_{\mathrm{T}} a_{\mathrm{cyl}}}
$$

Also this dimensionless value $\mathbf{F}$ can be used to characterize the locomotion. A similar value will result in a similar relative variation of the velocity.

Equation 10 together with Equation 8 provide a stronger statement for scaling and characterizing a system than Equation 11. These equations can be used for dimensioning the system.
In the keeping velocity phase, $L_{\mathrm{C}} / L_{\mathrm{T}}$, Equation 8 , defines how efficient the ballistic mode is. Assuming that the dimensioning of the cylinder and thus $F_{\text {cylN }}$ is defined by other load cases, like acceleration or applying a force at slow motion, then the used energy of a conventional cylinder is $E_{\mathrm{CC}}=$ $F_{\text {cylN }} L$. The energy not used for the motion is dissipated in the valve. In the ballistic mode during the keeping velocity phase the used energy is:

$$
E=F_{\text {cylN }} L \frac{L_{\mathrm{T}}+L_{\mathrm{R}}}{L_{\mathrm{C}}}
$$

whereby $L_{\mathrm{R}}$ is the stroke of the retraction including disengaging and engaging. $L_{\mathrm{R}}$ may be suppressed in this equation if the retraction is powered by a low pressure flow source, e.g. an accumulator loaded with the tank flow during the thrusts. In the simulated example is $L_{\mathrm{R}}=L_{\mathrm{T}}+L_{\text {overhead ED }} \approx L_{\mathrm{T}}$, whereby $L_{\text {overhead ED }}$ is the additional distance the cylinder moves while engaging and disengaging. By comparing the energy used by a conventional cylinder and HILA in ballistic mode, the energy consumption factor $e_{\mathrm{B}}$ can be defined:

$$
\begin{aligned}
e_{\mathrm{B}} & =\frac{L_{\mathrm{T}}+L_{\mathrm{R}}}{L_{\mathrm{C}}}=\frac{1+\frac{L_{\mathrm{R}}}{L_{\mathrm{T}}}}{\frac{F_{\mathrm{cyl}}}{F_{\mathrm{LX}}}} \\
& \approx 2 \frac{F_{\mathrm{LX}}}{F_{\text {cyl }}}
\end{aligned}
$$

For the limit case $F_{\mathrm{LX}} \approx F_{\text {cyl }}$, which can not be realized, is $e_{\mathrm{B}}=2$ and HILA in the ballistic mode would use approximately twice the energy of a conventional cylinder. The breakeven point is at $F_{\mathrm{LX}} \approx \frac{1}{2} F_{\text {cyl }}$, where the distance of the ballistic distance $L_{\mathrm{B}}$ is approximately the equal to the thrust stroke length $L_{\mathrm{T}}$. For the case $F_{\mathrm{LX}} \ll F_{\text {cyl }}$ the energy consumption factor $e_{\mathrm{B}}$ approachs zero as there is nearly no energy used in the ballistic mode.

The system's implementation, Figures 6 and 7, defines a quasi-constant duration for retracting, disengaging and engaging which in sum defines a lower limit for $T_{\mathrm{B}}, T_{\mathrm{B}} \geq T_{\mathrm{B} \text { limit }}=$ $T_{\mathrm{R}} . T_{\mathrm{B}}$ is the duration the load moves autonomously between two thrusts. $L_{\mathrm{B}}$, the distance the load moves between two thrusts, can be estimated by:

$$
L_{\mathrm{B}}=v_{0} T_{\mathrm{B}}+\frac{F_{\mathrm{LX}}}{m} \frac{T_{\mathrm{B}}^{2}}{2}
$$

This results in following condition for the load force.

$$
\begin{aligned}
F_{\mathrm{LX}} & <\frac{F_{\text {cyl }}}{1+\frac{v_{0} T_{\mathrm{B} \text { limit }}+\frac{F_{\mathrm{LX}}}{m} \frac{T_{\mathrm{B} \text { limit }}^{2}}{2}}{L_{\mathrm{T}}}} \\
& \approx \frac{F_{\text {cyl }}}{1+\frac{v_{0} T_{\mathrm{B} \text { limit }}}{L_{\mathrm{T}}}}
\end{aligned}
$$

The simplifications made lead to that also a load force near this limit may not be valid, but of the relevant case of small $r$, Equation 10, and high velocities $v_{0}$, this is a valuable condition.

In an application, the relative variation $r$, Equation 10, and absolute variation $\Delta v$, Equation 9, of the velocity has to be 
reasonable small. Equations 5 and 9 lead to following condition limiting the absolute variation in velocity:

$$
m_{L}>\frac{2 F_{\text {cyl }} L_{\mathrm{T}}}{v_{1}^{2}-v_{0}^{2}} \approx \frac{2 F_{\text {cyl }} L_{\mathrm{T}}}{\widehat{\Delta v}^{2}}
$$

The approximation is applicable for small $r$ and thus high velocities. A condition to limit the relative variation in velocity is derived from Equation 10b:

$$
m_{L}>\frac{2 F_{\text {cyl }} L_{\mathrm{T}}}{(\widehat{r}+1)^{2}-1} \frac{1}{v_{0}^{2}}
$$

\subsection{Control Inputs}

In the presented gait the system has the following control inputs which can be used to control the motion and to adjust to changing load conditions. The inputs can be used in combination to achieve optimal performance and efficiency.

\subsubsection{When to trigger the next driving stroke}

This control input is used for velocity control in the simulation, where the results are shown in Figures 8 and 9. The velocity control is simply realized by the state wait, Figure 4, by waiting for the velocity of the actuator to drop below a specified limit, before triggering the next thrust. Alternatively the duration between two thrusts can be used as control input which is commonly known as Pulse-Frequency Modulation (PFM).

\subsubsection{Force direction}

By choosing the cylinder chamber to connect to tank pressure while driving the load, the direction of the force is selected. In the simulated example that is used for accelerating, chamber $\mathrm{B}$, and braking, chamber $\mathrm{A}$.

Extending this concept, more than two different supply pressure levels can be utilized.

\subsubsection{Stroke length, when to disengage}

The energy delivered during a thrust depends on the stroke length, Equation 1. The stroke can be varied from zero to the stroke length of the cylinder considering margins for engaging and disengaging. In the case of a constant thrust frequency this control method is commonly known as Pulse-Width Modulation.

Stroke length variation is effective and efficient control input. The system's efficiency is only minimally reduced, see Equation 13. An advantage is that it also reduces the relative variation $r$, Equation 10, and absolute variation $\Delta v$, Equation 9, of the velocity, i.e. it makes the trajectory smoother.

\subsubsection{Valve opening}

In case of system P, Figure 6, the valves can be used to throttle the flow and thus reduce the cylinder force. This adds losses to the system but allows intervention during a thrust.

\subsubsection{Switching}

As in system D the necessary valves are already in place, the ballistic mode can be combined with hydraulic switching technology, see for example the hydraulic buck converter [6].

\subsubsection{Supply pressure}

The energy delivered during a thrust depends on the cylinder force and thus the supply pressure, Equation 4. This method has similarities to load sensing systems.

This method reduces the efficiency of the actuator but may improve the system's efficiency. It reduces also the relative variation $r$, Equation 10, and absolute variation $\Delta v$, Equation 9 , of the velocity, i.e. it makes the trajectory smoother.

\subsubsection{Number of cylinders}

The presented concept uses only one cylinder. A second cylinder, or any number of cylinders, can be used in parallel to increase force and thus acceleration. Also cylinders with different piston areas can be combined. By selecting the pistons, the actuator adjusts to a varying load or varying acceleration requirements. This method is commonly known as Pulse-Code Modulation.

Alternatively, a second cylinder can be used also to increase the maximum frequency.

\section{Conclusion}

The presented ballistic gait for HILA is intended to be used exclusively or in combination with other gaits. One other gait is presented in [1], where alternatingly, one cylinder engages and drives the load while the other retracts, to provide a smooth motion. The ballistic gait is energy efficient and supports high velocities but the motion is bumpy.

The ballistic gait brings switching technology, see for example the hydraulic buck converter [6], into the hydromechanical world. It has the ability to effectively recuperate energy.

This gait is feasible for loads with sufficient high mass and sufficient low load force, as external forces and friction. It is well suited for horizontal motion of huge masses but less suited for lifting.

Fast engaging and disengaging is necessary as it limits the achievable velocity and reduces the efficiency

This contribution presents the results of simulations of two systems realizing the ballistic gait. The system is analyzed, presenting equations for estimates of the undesired variation in velocity, energy efficiency, scaling, system limitations and design estimations.

Various control inputs are presented and analyzed concerning undesired variation in velocity and energy efficiency. 


\section{Nomenclature}

\begin{tabular}{lll}
\hline \multicolumn{2}{l}{ Designation Denotation } & Unit \\
\hline$F_{\text {cyl }}$ & cylinder force & $\mathrm{N}$ \\
$F_{\mathrm{L}}$ & external load force & $\mathrm{N}$ \\
$F_{\mathrm{LX}}$ & $\begin{array}{l}\text { load force including friction } \\
\text { distance, the load moves autonom- }\end{array}$ & $\mathrm{N}$ \\
$L_{\mathrm{B}}$ & $\begin{array}{l}\text { ously between two thrusts } \\
\text { distance, the load moves during one }\end{array}$ & $\mathrm{m}$ \\
$L_{\mathrm{C}}$ & $\begin{array}{l}\text { cycle, } L_{\mathrm{C}}=L_{\mathrm{T}}+L_{\mathrm{B}} \\
\text { thrust stroke length }\end{array}$ & $\mathrm{m}$ \\
$L_{\mathrm{T}}$ & $\begin{array}{l}\text { pressure in cylinder chamber A } \\
p_{\mathrm{A}}\end{array}$ & $\mathrm{Pa}$ \\
$p_{\mathrm{B}}$ & $\begin{array}{l}\text { pressure in cylinder chamber B } \\
T_{\mathrm{B}}\end{array}$ & $\mathrm{Pa}$ \\
& $\begin{array}{l}\text { dusation, the load moves autonom- } \\
T_{\mathrm{T}}\end{array}$ & $\mathrm{s}$ \\
\hline
\end{tabular}

\section{References}

[1] Martin Hochwallner and Petter Krus. Motion Control Concepts for the Hydraulic Infinite Linear Actuator. In Proceedings of the 9th FPNI PHD Symposium on Fluid Power, 2016. ISBN: 978-0-7918-5047-3.

[2] Martin Hochwallner, Magnus Landberg, and Petter Krus. The Hydraulic Infinite Linear Actuator properties relevant for control. In Proceedings of the 10th International Fluid Power Conference (10. IFK), volume 3, pages 411-424, 2016. http://nbnresolving.de/urn:nbn:de:bsz:14-qucosa-200646.

[3] Magnus Landberg, Martin Hochwallner, and Petter Krus. Novel Linear Hydraulic Actuator. ASME/BATH 2015 Symposium on Fluid Power \& Motion, Chicago, United States, 2015.

[4] Website of NoMagic - Cameo Systems Modeler. http://www.nomagic.com/products/cameo-systemsmodeler.html, visited 2017-03-15.

[5] Matti Linjama, H-P Vihtanen, Ari Sipola, and Matti Vilenius. Secondary Controlled Multi-Chamber Hydraulic Cylinder. In The 11th Scandinavian International Conference on Fluid Power, SICFP09, Linköping, Sweden, 2009.

[6] Helmut Kogler. The Hydraulic Buck Converter - Conceptual Study and Experiments. PhD thesis, 2012. ISBN: 978-3990330593.

[7] Björn Eriksson and Jan-Ove Palmberg. Individual Metering Fluid Power Systems: Challenges and Opportunities. Proceedings of the Institution of Mechanical Engineers. Part I, Journal of Systems and Control Engineering, 225(12):196-211, 2011.

[8] Andreas Kugi. Non-linear Control Based on Physical Models: Electrical, Mechanical and Hydraulic Systems. Number 260 in Lecture Notes in Control and Information Sciences. Springer, 2000. ISBN: 99-0147115-X.
[9] Mohieddine Jelali and Andreas Kroll. Hydraulic Servosystems: Modelling, Identification and Control. Advances in Industrial Control. Springer, 2004. ISBN: 9781-4471-1123-8.

[10] Christoph Gradl and Rudolf Scheidl. Performance of an Energy Efficient Low Power Stepper Converter. Energies, 10(4):445, 2017.

[11] Heinz Gall and Kurt Senn. FreilaufventileAnsteuerungskonzept zur Energieeinsparung bei hydraulischen Linearantrieben. Olhydraulik und Pneumatik, 38(1):38-44, 1994.

[12] ETP Transmission AB. ETP-OCTOPUS - Datasheet for Octopus.

[13] Linz Center of Mechatronics GmbH - Hydraulic Drives. FSVi 4.1 Datasheet - Fast Switching Valve Technolog, 2016. http://www.lcm.at/.

[14] Bernd Winkler, Andreas Ploeckinger, and Rudolf Scheidl. A Novel Piloted Fast Switching Multi Poppet Valve. International Journal of Fluid Power, 11(3):714,2010 .

[15] John Watton. Fundamentals of Fluid Power Control. Cambridge University Press, Cambridge, UK New York, 2009. ISBN: 9780521762502.

[16] Wikipedia: Transition from walking to running. https://en.wikipedia.org/wiki/Transition_from_walking_to_running, visited 2017-05-18. 